\title{
Continuous-Time Mean-Variance Asset-Liability Management with Hidden Markovian Regime Switching
}

\author{
Ling Zhang \\ Department of Economic and Trade, Guangdong University of Finance, Guangzhou 510521, China \\ Correspondence should be addressed to Ling Zhang; zhang199@mail2.sysu.edu.cn
}

Received 13 February 2014; Accepted 22 May 2014; Published 15 June 2014

Academic Editor: Piermarco Cannarsa

Copyright ( 2014 Ling Zhang. This is an open access article distributed under the Creative Commons Attribution License, which permits unrestricted use, distribution, and reproduction in any medium, provided the original work is properly cited.

\begin{abstract}
This paper considers a continuous-time mean-variance asset-liability management problem with incompletely observable information. An investor can only observe the prices of the asset and liability and the dynamics of the unobservable states of the underlying financial market is described by a hidden Markovian chain. The price of the risky asset is assumed to be governed by a hidden Markovian regime switching geometric Brownian motion and the liability is assumed to follow a hidden Markovian regime switching Brownian motion with drift, respectively. The appreciation rates of the risky asset and the liability are modulated by the hidden Markovian chain. By using the separation principle, the filtering-estimation problem and the mean-variance assetliability management problem are discussed. The explicit expressions for the optimal asset-liability management strategy and the mean-variance efficient frontier are determined by using the stochastic maximum principle.
\end{abstract}

\section{Introduction}

Liability has a significant impact on asset allocation for many asset management institutions, like pension fund, bank, and insurance company. Asset-liability management problem has been receiving more and more attention, and the past decade has witnessed the increasing research on the assetliability management problem in the framework of meanvariance criterion presented by Markowitz [1]. Actually, the mean-variance asset-liability management problem is a portfolio optimization problem such that the expectation of the terminal surplus is maximized while the risk measured by the variance of the terminal surplus is minimized. Sharpe and Tint [2] first present a single-period mean-variance asset-liability management model and show that the optimal investment decision of a company should take its liabilities into account. Later, Kell and Müller [3] find that liabilities do have a significant effect on the efficient frontier. The role of liability has been studied by Leippold et al. [4] in a multiperiod setting by using the geometric approach and the embedding technique of $\mathrm{Li}$ and $\mathrm{Ng}$ [5]. Chiu and $\mathrm{Li}$ [6] generalize the mean-variance asset-liability management problem to a continuous-time setting and derive the analytical optimal portfolio strategy and the optimal initial funding ratio by using the stochastic LQ control. Xie and Li [7] consider the continuous-time mean-variance asset-liability management problem where the liability follows the Brownian motion with drift and derive the optimal strategy. Yi et al. [8] consider the impact of the uncertain exit time on the optimal asset-liability management strategy. Xie et al. [9] extend the model of Xie and $\mathrm{Li}$ [7] by assuming that the liability follows a geometric Brownian motion in a continuous-time incomplete market. Yao et al. [10] explore the effect of the endogenous liabilities in a financial market with only risky asset. The key assumption of the above mentioned papers is that the market parameters, such as the bank interest rate, the appreciation rate, and the volatility of the underlying assets and liabilities, are constants in the continuous-time setting and are an identical independent distribution in the discrete-time setting. However, from an economic practice perspective, it is more beneficial to develop an asset-liability management model that incorporates the stochastic evolution of the economic environment.

To catch the stochastic feature of the financial market, regime switching models have become prevalent in finance and economics over the last few years. The main feature of the regime switching models is that the states (or regimes) of the financial market are modeled by a Markovian chain and various parameters, such as the bank interest rate, 
the appreciation rates, and the volatilities of the underlying assets and liabilities, will be assigned different values under different market states. In view of the study of the mean-variance asset-liability management problem with Markovian regime switching, Chen et al. [11] extend the model of Xie et al. [9] by adding an independent Brownian motion in the evolution of the liability. They examine the feasibility of the asset-liability management problem in a continuous-time setting and derive the analytical optimal asset-liability management strategy by using the LQ control. By incorporating liability into the continuous-time meanvariance portfolio optimization problem in a Markovian regime switching market, Xie [12] derives the optimal assetliability management strategy by using the LQ technique and Lagrange multiplier technique. Chen and Yang [13] consider a multiperiod asset-liability management problem in the Markovian regime switching market and derive the analytical optimal investment strategy. Wei et al. [14] explore the time consistent optimal strategy of the mean-variance asset-liability management problem.

In the existing literature on the mean-variance assetliability management problem with Markovian regime switching, the states of the financial market are assumed to be completely observed at any time. Actually, an investor can only observe the prices of asset and liability rather than the whole information of the financial market. There still exist some states of the market that cannot be observed by the investor. In the financial market with incompletely observable states, hidden Markovian chain is the most frequently used model to describe the evolution of the unobservable market states. The portfolio optimization problem in the hidden Markovian regime switching financial market has been investigated by Sotomayor and Cadenillas [15], Çanakoğlu and Özekici [16], and Elliott et al. [17]. Comparing the existing literature, there is relatively little work on the meanvariance portfolio selection problem considering liability in the hidden Markovian regime switching financial market.

In this paper, a mean-variance asset-liability management problem is considered in a continuous-time hidden Markovian regime switching market with a risky asset and a riskfree asset. We assume that the dynamics of the unobservable market states follows a continuous-time finite-state hidden Markovian chain and the appreciation rates of the risky asset and the liability are modulated by the hidden Markovian chain. We suppose that the price of the risky asset is governed by a hidden Markovian regime switching geometric Brownian motion and the dynamics of the exogenous liability is described by a hidden Markovian regime switching Brownian motion with drift, respectively. We consider the situation that an investor wants to maximize the expectation of the terminal surplus of liability from wealth while minimizing the risk measured by the variance of the terminal surplus. By using the separation principle for filtering and control, the problem of parameters estimation and the mean-variance portfolio optimization problem are solved separately. The stochastic maximum principle is used to derive the explicit solution for the mean-variance portfolio optimization problem. And the mean-variance efficient frontier is also determined.
A brief outline of this paper is as follows. Section 2 presents the prices dynamics of the underlying assets and liability and describes the mean-variance asset-liability management problem in the hidden Markovian regime switching market. Section 3 demonstrates the use of the separation principle for filtering and control to separate filtering-estimation problem and the mean-variance asset-liability management problem. The optimal strategy of the auxiliary problem is derived in Section 4. The explicit optimal investment strategy and the efficient frontier for the mean-variance asset-liability management problem are presented in Section 5. Two special cases are given in Section 6. Section 7 gives some concluding remarks.

\section{Hidden Markovian Model with Liability}

Suppose that there exist one risk-free asset and one risky asset in the financial market where these assets can be traded continuously over time within a finite-time horizon $[0, T]$, where $T \leq \infty$. We denote by $(\Omega, \mathscr{F}, \mathscr{P})$ the complete probability space equipped with the filtration $\mathbb{F}=\left(\mathscr{F}_{t}\right)_{t \geq 0}$ and $\left\{\left(B^{S}(t), B^{L}(t)\right): t \in[0, T]\right\}$ is a given two-dimensional standard Brownian motion defined on $(\Omega, \mathscr{F}, \mathscr{P})$.

2.1. The Evolution of the Unobservable Market States. Suppose that $X=\{X(t): t \in[0, T]\}$ is a continuous-time stationary hidden Markovian process on $(\Omega, \mathscr{F}, \mathscr{P})$ with finite-state space $\mathscr{M}=\left\{e_{1}, \ldots, e_{m}\right\}$, where $e_{i}=(0, \ldots, 1, \ldots, 0)^{\prime} \in \mathbb{R}^{m}$ and has the generator $Q=\left(q_{i j}\right)_{m \times m}$. Here, $q_{i j}$ is a constant intensity representing the transition of process $X$ from state $e_{j}$ to state $e_{i}$ for each $i, j=1,2, \ldots, m$ and satisfies $q_{i j} \geq 0$ for $i \neq j$ and $\sum_{i=1}^{m} q_{i j}=0$. Since $X$ is a hidden Markovian chain, it means that the states of $X$ cannot be observed directly in the financial market. Following Elliott et al. [17], we assume that the dynamics of the hidden Markovian process $X$ satisfies the following semimartingale presentation:

$$
X(t)=X_{0}+\int_{0}^{t} Q X(s) d s+M(t),
$$

where $X_{0}$ is the initial unobservable market state at time 0 and $\{M(t): t \in[0, T]\}$ is an $\mathbb{R}^{m}$-valued martingale in the sense of the filtration generated by $X$ with respect to $\mathscr{P}$.

2.2. The Prices Dynamics of Assets and Liability. We consider a financial market with one risk-free asset and one risky asset. Let $r \geq 0$ denote the constant interest rate of the risk-free asset and let $P(t)$ denote the price of the risk-free asset at time $t$ which is described by the following ordinary differential equation (ODE):

$$
d P(t)=r P(t) d t, \quad P(0)=p_{0}, \quad t \in[0, T] .
$$

The price of the risky asset at time $t$ is denoted by $S(t)$ and the corresponding price process $S=\{S(t), 0 \leq t \leq$ $T\}$ evolves as the following hidden Markovian-modulated stochastic differential equation (SDE):

$$
d S(t)=\mu(t, X(t)) S(t) d t+\sigma_{S} S(t) d B^{S}(t), \quad t \in[0, T]
$$


where $\mu(t, X(t))$ is the appreciation rate of the risky asset at time $t$ when the state of the financial market at time $t$ is $X(t)$ and $\sigma_{S}>0$ is the constant volatility of the risky asset. Suppose that process $X$ is independent of the Brownian motion $B^{S}=$ $\left\{B^{S}(t), t \in[0, T]\right\}$.

During the process of investment, the investor has to withdraw some money continuously to pay for liability. Let $L(t)$ denote the accumulative exogenous liability at time $t$ and the evolution of the liability process $L=\{L(t), t \in[0, T]\}$ is governed by the following hidden Markovian-modulated stochastic differential equation (SDE):

$$
d L(t)=\vartheta(t, X(t)) d t+\sigma_{L} d B^{L}(t), \quad t \in[0, T],
$$

where $\vartheta(t, X(t))$ is the appreciation rate of the liability at time $t$ when the state of the financial market at time $t$ is $X(t), \sigma_{L}>0$ is the constant volatility of this liability, and $B^{L}=\left\{B^{L}(t), t \in[0, T]\right\}$ is a one-dimensional standard Brownian motion which is independent of $X$ and $B^{S}$. Since the appreciation rates in (3) and (4) are modulated by the unobservable market states $X$, we assume that $\mu, \vartheta$ are unobservable to the investor. Actually, the Brownian motion $B^{S}$ and $B^{L}$ also cannot be observed by the investor. So, the investor can only obtain the price information of $P, S$, and $L$ and make the optimal investment decision just based on the observed price information $(P, S, L)$ up to that moment.

2.3. Wealth Process. Suppose that the trading of assets occurs in a self-financing way (i.e., there is no consumption and there is no income) and there is no other transaction cost or fee. At each moment, the investor determines the money invested in the risky asset based on all of the observed information up to that moment. We denote by $\theta(t)$ the amount invested in the risky asset at time $t$ and by $Z^{\theta}(t)$ the net wealth of the investor at time $t$ corresponding to $\theta$. For notation simplicity, we suppress the superscript $\theta$ and write $Z(t)$ for $Z^{\theta}(t)$ in this paper. Let $h^{0}(t)$ and $h(t)$ denote the number of shares of the risk-free asset and the risky asset held by the investor at time $t$, respectively. The $\theta(t)=h(t) S(t)$ and $Z(t)-\theta(t)=$ $h^{0}(t) P(t)$ are the amount of money invested in the risky asset and the risk-free asset at time $t$, respectively.

If the trading of assets occurs at time point $t$ and there is no withdrawal of money during the time interval $(t, t+\Delta t)$, then the increment of wealth at time $t+\Delta t$ is given by

$$
\begin{aligned}
Z(t+\Delta t)-Z(t)= & h^{0}(t)(P(t+\Delta t)-P(t)) \\
& +h(t)(S(t+\Delta t)-S(t)) .
\end{aligned}
$$

If there exists money withdrawal with the amount $L(t+\Delta t)-$ $L(t)$ at time $t+\Delta t$ to pay for the liability, then (5) should be replaced by

$$
\begin{aligned}
Z(t+ & \Delta t)-Z(t) \\
= & h^{0}(t)(P(t+\Delta t)-P(t))+h(t) \\
& \times(S(t+\Delta t)-S(t))-(L(t+\Delta t)-L(t)),
\end{aligned}
$$

for $0 \leq t \leq T$. Letting $\Delta t \rightarrow 0^{+}$, the dynamics of net wealth in a continuous-time analogue is described as follows:

$$
d Z(t)=h^{0}(t) d P(t)+h(t) d S(t)-d L(t), \quad 0 \leq t \leq T .
$$

Substituting (2)-(4) into (7), the evolution of the net wealth process $Z$ over time is specified as follows:

$$
\begin{gathered}
d Z(t)=[r Z(t)+\theta(t)(\mu(t, X(t))-r)-\vartheta(t, X(t))] d t \\
+\theta(t) \sigma_{S} d B^{S}(t)-\sigma_{L} d B^{L}(t), \\
Z(0)=z_{0},
\end{gathered}
$$

where $z_{0}$ is the initial net wealth at time 0 .

Definition 1. A portfolio strategy $\theta(\cdot)$ is called an admissible strategy if $\theta(\cdot) \in L_{\mathscr{F}}^{2}([0, T] ; \mathbb{R})$ and (8) has a unique solution. We refer to $\mathscr{A}\left(z_{0}, X_{0}\right)$ as the set of the admissible strategy with the net initial wealth $z_{0}$ and the initial market state $X_{0}$.

2.4. Asset-Liability Management Problem. The objective of an investor is to find an optimal asset-liability management strategy such that the expected net terminal wealth of $Z(T)$, $\mathbb{E}(Z(T))$, is maximized and the risk, measured by the variance of the net terminal wealth $\operatorname{Var}(Z(T))$, is minimized. Then, the mean-variance asset-liability management problem can be described as follows:

$$
\begin{aligned}
& \max _{\theta \in \in \mathscr{A}\left(z_{0}, X_{0}\right)} \mathbb{E}(Z(T))-\omega \operatorname{Var}(Z(T)), \\
& \text { s.t. } \quad d Z(t) \\
&=[r Z(t)+\theta(t)(\mu(t, X(t))-r)-\vartheta(t, X(t))] d t \\
& \quad+\theta(t) \sigma_{S} d B^{S}(t)-\sigma_{L} d B^{L}(t),
\end{aligned}
$$

with $\omega>0$ representing the investor's trade-off between the expected return and the risk.

An admissible strategy $\theta^{*}$ is called mean-variance efficient if there is no other admissible strategy $\theta$ such that $\mathbb{E}(Z(T)) \geq \mathbb{E}\left(Z^{*}(T)\right)$ and $\operatorname{Var}(Z(T)) \leq \operatorname{Var}\left(Z^{*}(T)\right)$ with at least one inequality holding strictly, where $Z(T)$ and $Z^{*}(T)$ are the terminal net wealth corresponding to the admissible strategy $\theta$ and $\theta^{*}$. And the pair $\left(\mathbb{E}\left(Z^{*}(T)\right)\right.$, $\left.\operatorname{Var}\left(Z^{*}(T)\right)\right)$ corresponding to the efficient strategy $\theta^{*}$ is called an efficient point. The set of all efficient points is called the efficient frontier.

Notice that $\mu, \mathcal{\vartheta}, B^{S}$, and $B^{L}$ are unobservable parameters in the evolution of the net wealth in problem (9). It means that the investor confronts an asset-liability management problem with incomplete observations. Following Elliott et al. [17], problem (9) can be decomposed into two separable problems: a filtering-estimation problem in which the investor infers the unobservable model parameters based on the observed information up to that moment and an optimization problem in which the investor uses the current estimates of the unobservable model parameters to determine the optimal asset-liability management strategy. 


\section{Separation Principle}

The separation principle is a significant technique to deal with the stochastic optimal control problem with incompletely observable information. As shown in Detemple [18, 19], Dothan and Feldman [20], and Gennote [21], according to the separation principle, the asset-liability management problem (9) can be decomposed into two separate problems: a filtering-estimation problem in which the investor estimates the current values of the unobservable parameters, $\bar{\mu}(t)$ and $\bar{\vartheta}(t)$, by using the Kalman filtering method and an optimization problem in which the investor uses his current estimate $\bar{\mu}(t)$ and $\bar{\vartheta}(t)$ to make an optimal asset-liability management decision. Although the appreciation rates $\mu(t)$ and $\mathcal{\vartheta}(t)$ are unknown, the separation principle assures that the assetliability management problem (9) can be solved on the basis of its estimate values.

Let $\mathscr{I}=\{\mathscr{I}(t)=(P(t), S(t), L(t)), t \in[0, T]\}$ denote the joint observed information process. Let $\mathscr{F}^{\mathscr{I}}=\left\{\mathscr{F}_{t}^{\mathscr{I}}, t \in\right.$ $[0, T]\}$ and $\mathscr{F}^{X}=\left\{\mathscr{F}_{t}^{X}, t \in[0, T]\right\}$ denote the right continuous, complete filtration generated by the observed information process $\mathscr{I}$ and the unobservable market states process $X$, respectively. Then $\mathscr{F}^{\mathscr{I}}$ and $\mathscr{F}^{X}$ summarize the structure of the observed market information and the hidden-marketstate information, respectively. Furthermore, we denote by $\mathscr{G}=\left\{\mathscr{G}_{t}, \mathscr{G}_{t}=\mathscr{F}_{t}^{\mathscr{I}} \vee \mathscr{F}_{t}^{X}, t \in[0, T]\right\}$ the full information of the financial market.

For any integrable $\mathscr{G}$-adapted process $g=\{g(t), t \in$ $[0, T]\}$, let $\bar{g}(t)=E\left[g(t) \mid \mathscr{F}_{t}^{\mathscr{I}}\right]$. We define an $\mathscr{F}^{\mathscr{F}}$-adapted innovation process $\bar{B}=\left\{\left(\bar{B}^{S}(t), \bar{B}^{L}(t)\right), t \in[0, T]\right\}$ on the probability space $(\Omega, \mathscr{F}, \mathscr{P})$ which is given as follows:

$$
\begin{aligned}
& d \bar{B}^{S}(t)=\frac{\mu(t, X(t))-\bar{\mu}(t)}{\sigma_{S}} d t+d B^{S}(t), \\
& d \bar{B}^{L}(t)=\frac{\vartheta(t, X(t))-\bar{\vartheta}(t)}{\sigma_{L}} d t+d B^{L}(t),
\end{aligned}
$$

where $\bar{\mu}(t)=E\left[\mu(t, X(t)) \mid \mathscr{F}_{t}^{\mathscr{I}}\right]$ and $\bar{\vartheta}(t)=E[\vartheta(t, X(t)) \mid$ $\left.\mathscr{F}_{t}^{\mathscr{F}}\right]$ for each $t \in[0, T]$. Following Lipster and Shiryaev [22], the $\mathscr{P}$-augmentation of the natural filtration generated by $\bar{B}=$ $\left\{\left(\bar{B}^{S}(t), \bar{B}^{L}(t)\right), t \in[0, T]\right\}$ coincides with the filtration $\mathscr{F}^{\mathscr{I}}$. And the innovation process $\bar{B}=\left\{\left(\bar{B}^{S}(t), \bar{B}^{L}(t)\right), t \in[0, T]\right\}$ is a Brownian motion with respect to the investor's information filtration $\mathscr{F}^{\mathscr{I}}$. Correspondingly, the dynamics of the risky asset price in terms of $\bar{B} S(t)$ can now be rewritten as

$$
d S(t)=S(t) \bar{\mu}(t) d t+\sigma_{S} S(t) d \bar{B}^{S}(t),
$$

and the dynamics of the liability with respect to $\bar{B}^{L}(t)$ can be rewritten as

$$
d L(t)=\bar{\vartheta}(t) d t+\sigma_{L} d \bar{B}^{L}(t) .
$$

Furthermore, the dynamics of the net wealth process changes into

$$
\begin{aligned}
d Z(t)= & {[r Z(t)+\theta(t)(\bar{\mu}(t)-r)-\bar{\vartheta}(t)] d t } \\
& +\theta(t) \sigma_{S} d \bar{B}^{S}(t)-\sigma_{L} d \bar{B}^{L}(t) .
\end{aligned}
$$

Notice that processes (11)-(13) are all $\mathscr{F}_{t}^{\mathscr{I}}$-adapted, and $\mathscr{F}_{t}^{\mathscr{I}}$ is generated by the observable information of the assets and liability prices up to time $t$. Since the investor has access to the price filtration $\mathscr{F}_{t}^{\mathscr{I}}$, the asset-liability management problem with incompletely observable information (9) can now be transformed into the corresponding optimization problem with completely observable information. Then, the original mean-variance asset-liability management problem (9) can be transformed into the following optimization problem with complete observation:

$$
\begin{aligned}
& \max _{\theta \in \mathscr{A}\left(z_{0}, X_{0}\right)} \mathbb{E}(Z(T))-\omega \operatorname{Var}(Z(T)), \\
& \text { s.t. } \quad d Z(t)= {[r Z(t)+\theta(t)(\bar{\mu}(t)-r)-\bar{\vartheta}(t)] d t } \\
&+\theta(t) \sigma_{S} d \bar{B}_{t}^{S}-\sigma_{L} d \bar{B}_{t}^{L} .
\end{aligned}
$$

With the estimated parameters, the investor can determine the optimal asset-liability management strategy of problem (14) by using the technique of stochastic optimal control.

\section{The Auxiliary Problem}

To determine the optimal solution for problem (14), we first introduce the following auxiliary problem:

$$
\begin{aligned}
\max _{\theta \in \mathscr{A}\left(z_{0}, X_{0}\right)} \mathbb{E}\left(\lambda Z(T)-\omega Z(T)^{2}\right), \\
\text { s.t. } d Z(t)=[r Z(t)+\theta(t)(\bar{\mu}(t)-r)-\bar{\vartheta}(t)] d t \\
+\theta(t) \sigma_{S} d \bar{B}^{S}(t)-\sigma_{L} d \bar{B}^{L}(t),
\end{aligned}
$$

where $\lambda \in \mathbb{R}$ is a pregiven parameter.

Denote

$\widehat{\Pi}(\lambda, \omega)$

$=\{\theta \mid \theta$ is an optimal solution of problem (15) $\}$,

$\Pi(\omega)$

$=\{\theta \mid \theta$ is an optimal solution of problem (14) $\}$.

Following Zhou and Li [23], the relationship between the optimal solutions for problem (14) and problem (15) is given by the following lemma. Its proof is similar to Zhou and $\mathrm{Li}$ [23] and is omitted here.

Lemma 2. For any $\omega>0$, one has $\Pi(\omega) \subseteq \bigcup_{\lambda \in \mathbb{R}} \widehat{\Pi}(\lambda, \omega)$. If $\theta^{*} \in \Pi(\omega)$, then $\theta^{*} \in \widehat{\Pi}\left(\lambda^{*}, \omega\right)$, where $\lambda^{*}=1+\left.2 \omega \mathbb{E}(Z(T))\right|_{\theta^{*}}$. Furthermore, if $\theta^{*} \in \widehat{\Pi}\left(\lambda^{*}, \omega\right)$, a necessary condition for $\theta^{*} \in$ $\Pi(\omega)$ is $\lambda^{*}=1+\left.2 \omega \mathbb{E}(Z(T))\right|_{\theta^{*}}$.

Note that the objective of problem (15) can be written as

$$
\mathbb{E}\left(\lambda Z(T)-\omega Z(T)^{2}\right)=\mathbb{E}\left[-\omega\left(Z(T)-\frac{\lambda}{2 \omega}\right)^{2}+\frac{\lambda^{2}}{4 \omega}\right] .
$$

The term $\lambda^{2} / 4 \omega$ is a constant and it has no impact on the optimal solution for problem (15). So it can be ignored in 
the objective function of problem (15). Let $\gamma=\lambda / 2 \omega$; then the auxiliary problem (15) can be equivalently rewritten as the following stochastic control problem:

$$
\begin{aligned}
& \max _{\theta \in \mathscr{A}\left(z_{0}, X_{0}\right)} \mathbb{E}\left[-\frac{1}{2} \omega(Z(T)-\gamma)^{2}\right], \\
& \text { s.t. } \quad d Z(t)=[r Z(t)+\theta(t)(\bar{\mu}(t)-r)-\bar{\vartheta}(t)] d t \\
&+ \theta(t) \sigma_{S} d \bar{B}^{S}(t)-\sigma_{L} d \bar{B}^{L}(t) .
\end{aligned}
$$

Using the stochastic maximum principle, we can determine the optimal solution for the optimization problem (18) and the results are summarized by the following theorem.

Theorem 3. For problem (18), the optimal solution is given by

$$
\theta^{*}(t)=\frac{\tau(t)}{\sigma_{S}}\left(Z^{*}(t)+\kappa(t)\right),
$$

where $Z^{*}(t)$ is the net wealth at time $t$ under the optimal strategy $\theta^{*}(t)$ for $t \in[0, T]$, and

$$
\begin{aligned}
& \kappa(t)=-e^{-r(T-t)}\left(\gamma+\int_{t}^{T} \bar{\vartheta}(s) e^{r(T-s)} d s\right), \\
& \tau(t)=\frac{r-\bar{\mu}(t)}{\sigma_{S}} .
\end{aligned}
$$

Proof. For problem (18), the Hamiltonian is given by

$$
\begin{aligned}
& H\left(t, \theta(t), Z(t), \varphi(t), \psi_{1}(t), \psi_{2}(t)\right) \\
&= {[r Z(t)+\theta(t)(\bar{\mu}(t)-r)-\bar{\vartheta}(t)] \varphi(t) } \\
&+\sigma_{S} \theta(t) \psi_{1}(t)-\sigma_{L} \psi_{2}(t),
\end{aligned}
$$

along with the adjoint equation given as

$$
\begin{aligned}
d \varphi(t) & =-r \varphi(t) d t+\psi_{1}(t) d \bar{B}^{S}(t)+\psi_{2}(t) d \bar{B}^{L}(t), \\
\varphi(T) & =-\omega Z(T)+\omega \gamma .
\end{aligned}
$$

We consider a solution of (22) as

$$
\varphi(t)=a(t) Z(t)+b(t),
$$

where $a(t)$ and $b(t)$ are both deterministic first-order continuously differential functions with respect to $t \in[0, T]$. Applying Itô lemma to (23), we have

$$
\begin{aligned}
d \varphi(t)=\{ & \dot{a}(t) Z(t)+a(t)[r Z(t)+(\bar{\mu}(t)-r) \theta(t)-\bar{\vartheta}(t)] \\
& +\dot{b}(t)\} d t+\sigma_{S} a(t) \theta(t) d \bar{B}^{S}(t)-a(t) \sigma_{L} d \bar{B}^{L}(t) .
\end{aligned}
$$

Substituting (23) into the adjoint equation (22) introduces

$$
\begin{aligned}
d \varphi(t)= & -r(a(t) Z(t)+b(t)) d t \\
& +\psi_{1}(t) d \bar{B}^{S}(t)+\psi_{2}(t) d \bar{B}^{L}(t) .
\end{aligned}
$$

Comparing (24) with (25), we have

$$
\begin{gathered}
-r(a(t) Z(t)+b(t)) \\
=a(t)[r Z(t)+(\bar{\mu}(t)-r) \theta(t)-\bar{\vartheta}(t)] \\
+\dot{a}(t) Z(t)+\dot{b}(t), \\
\psi_{1}(t)=\sigma_{S} a(t) \theta(t), \\
\psi_{2}(t)=-\sigma_{L} a(t) .
\end{gathered}
$$

We assume that $\theta^{*}(t)$ is the candidate optimal strategy for the Hamiltonian (21), $Z^{*}(t)$ is the wealth under the candidate optimal strategy $\theta^{*}(t)$, and $\left(\varphi^{*}(t), \psi_{1}^{*}(t), \psi_{2}^{*}(t)\right)$ is the corresponding solution of the adjoint equation (22). Then, we have

$$
\begin{aligned}
H( & \left.t, \theta^{*}(t), Z^{*}(t), \varphi^{*}(t), \psi_{1}^{*}(t), \psi_{2}^{*}(t)\right) \\
= & {\left[r Z^{*}(t)+\theta^{*}(t)(\bar{\mu}(t)-r)-\bar{\vartheta}(t)\right] \varphi^{*}(t) } \\
& +\sigma_{S} \theta^{*}(t) \psi_{1}^{*}(t)-\sigma_{L} \psi_{2}^{*}(t) \\
= & \left(r Z^{*}(t)-\bar{\vartheta}(t)\right) \varphi^{*}(t)-\sigma_{L} \psi_{2}^{*}(t) \\
& +\theta^{*}(t)\left[(\bar{\mu}(t)-r) \varphi^{*}(t)+\sigma_{S} \psi_{1}^{*}(t)\right] .
\end{aligned}
$$

Since the Hamiltonian (21) is a linear function of the candidate optimal control $\theta^{*}(t)$, it is easy to get the conclusion that the coefficient of $\theta^{*}(t)$ in (29) must be equal to zero; that is,

$$
(\bar{\mu}(t)-r) \varphi^{*}(t)+\sigma_{S} \psi_{1}^{*}(t)=0 .
$$

Substituting (23) and (27) into (30), we have

$$
\begin{aligned}
\theta^{*}(t)=\frac{r-\bar{\mu}(t)}{a(t) \sigma_{S}^{2}} \varphi^{*}(t) & =\frac{r-\bar{\mu}(t)}{a(t) \sigma_{S}^{2}}\left(a(t) Z^{*}(t)+b(t)\right) \\
& =\frac{\tau(t)}{\sigma_{S}}\left(Z^{*}(t)+\kappa(t)\right),
\end{aligned}
$$

where $\tau(t)=(r-\bar{\mu}(t)) / \sigma_{S}$ and $\kappa(t)=b(t) / a(t)$.

On the other hand, (26) implies

$$
\begin{aligned}
\theta^{*}(t)= & \left(\dot{a}(t) Z^{*}(t)+2 r a(t) Z^{*}(t)-a(t) \bar{\vartheta}(t)\right. \\
& +\dot{b}(t)+r b(t)) \times(a(t)(r-\bar{\mu}(t)))^{-1} .
\end{aligned}
$$

Comparing (31) and (32) results in

$$
\begin{gathered}
\dot{a}(t)+\left(2 r-\tau(t)^{2}\right) a(t)=0, \\
a(T)=-\omega, \\
\dot{b}(t)+\left(r-\tau(t)^{2}\right) b(t)-\overline{9}(t) a(t)=0, \\
b(T)=\omega \gamma .
\end{gathered}
$$

Solving the ODEs (33), the solutions are given as follows:

$$
\begin{aligned}
& a(t)=-\omega e^{\int_{t}^{T}\left(2 r-\tau(t)^{2}\right) d s} \\
& b(t)=\omega e^{\int_{t}^{T}\left(r-\tau(t)^{2}\right) d s}\left[\gamma+\int_{t}^{T} \bar{\vartheta}(s) e^{r(T-s)} d s\right] .
\end{aligned}
$$


Then

$$
\kappa(t)=-e^{-r(T-t)}\left[\gamma+\int_{t}^{T} \bar{\vartheta}(s) e^{r(T-s)} d s\right] .
$$

Hence, the proof is completed.

Substituting (31) and (34) into (26)-(28), we can obtain the solutions for the adjoint equation given as follows:

$$
\begin{aligned}
& \varphi^{*}(t)=- \omega e^{\int_{t}^{T}\left(2 r-\tau(t)^{2}\right) d s} \\
& \times\left[Z^{*}(t)-e^{-r(T-t)}\left(\gamma+\int_{t}^{T} \overline{\mathcal{Y}}(s) e^{r(T-s)} d s\right)\right], \\
& \psi_{1}^{*}(t)=- \omega \tau(t) e^{\int_{t}^{T}\left(2 r-\tau(t)^{2}\right) d s} \\
& \times {\left[Z^{*}(t)-e^{-r(T-t)}\left(\gamma+\int_{t}^{T} \overline{\mathcal{9}}(s) e^{r(T-s)} d s\right)\right], } \\
& \psi_{2}^{*}(t)=\omega \sigma_{L} e^{\int_{t}^{T}\left(2 r-\tau(t)^{2}\right) d s} .
\end{aligned}
$$

Substituting the optimal control (19) into the dynamics of the net wealth (13) results in

$$
\begin{gathered}
d Z^{*}(t)=\left[\left(r-\tau(t)^{2}\right) Z^{*}(t)-\tau(t)^{2} \kappa(t)-\bar{\vartheta}(t)\right] d t \\
+\tau(t)\left(Z^{*}(t)+\kappa(t)\right) d \bar{B}^{S}(t)-\sigma_{L} d \bar{B}^{L}(t), \\
Z^{*}(0)=z_{0} .
\end{gathered}
$$

Applying Itô lemma to $\left(Z^{*}(t)\right)^{2}$ gives

$$
\begin{gathered}
d\left(Z^{*}(t)\right)^{2}=\left[\left(2 r-\tau(t)^{2}\right)\left(Z^{*}(t)\right)^{2}-2 \bar{\vartheta}(t) Z^{*}(t)\right. \\
\left.+\tau(t)^{2} \kappa(t)^{2}+\sigma_{L}^{2}\right] d t+2 Z^{*}(t) \\
\times\left[\tau(t)\left(Z^{*}(t)+\kappa(t)\right) d \bar{B}^{S}(t)-\sigma_{L} d \bar{B}^{L}(t)\right] \\
\left(Z^{*}(0)\right)^{2}=\left(z_{0}\right)^{2} .
\end{gathered}
$$

Taking expectations on both (37) and (38), we have

$$
\begin{gathered}
d \mathbb{E}\left(Z^{*}(t)\right) \\
=\left[\left(r-\tau(t)^{2}\right) \mathbb{E}\left(Z^{*}(t)\right)-\tau(t)^{2} \kappa(t)-\bar{\vartheta}(t)\right] d t, \\
\mathbb{E}\left(Z^{*}(0)\right)=z_{0} . \\
d \mathbb{E}\left(Z^{*}(t)\right)^{2} \\
=\left[\left(2 r-\tau(t)^{2}\right) \mathbb{E}\left(Z^{*}(t)\right)^{2}\right. \\
\left.-2 \bar{\vartheta}(t) \mathbb{E}\left(Z^{*}(t)\right)+\tau(t)^{2} \kappa(t)^{2}+\sigma_{L}^{2}\right] d t, \\
\mathbb{E}\left(Z^{*}(0)\right)^{2}=\left(z_{0}\right)^{2} .
\end{gathered}
$$

Solving (39), we have

$$
\begin{aligned}
\mathbb{E}\left(Z^{*}(t)\right)= & z_{0} e^{\int_{0}^{t}\left(r-\tau(s)^{2}\right) d s} \\
& -\int_{0}^{t}\left(\tau(s)^{2} \kappa(s)+\bar{\vartheta}(s)\right) e^{\int_{s}^{t}\left(r-\tau(k)^{2}\right) d k} d s \\
= & \gamma \alpha(t)+\beta(t), \\
& \mathbb{E}\left(Z^{*}(T)\right)=\gamma \alpha+\beta,
\end{aligned}
$$

where

$$
\begin{gathered}
\alpha(t)=e^{-r(T-t)}\left(1-e^{-\int_{0}^{t} \tau(s)^{2} d s}\right), \\
\beta(t)=e^{\int_{0}^{t}\left(r-\tau(s)^{2}\right) d s}\left(z_{0}-\int_{0}^{T} \bar{\vartheta}(t) e^{-r t} d t\right) \\
+e^{r t} \int_{t}^{T} \bar{\vartheta}(s) e^{-r s} d s \\
\alpha=\alpha(T)=1-e^{-\int_{0}^{T} \tau(t)^{2} d t}, \\
\beta=\beta(T)=e^{\int_{0}^{T}\left(r-\tau(t)^{2}\right) d t}\left(z_{0}-\int_{0}^{T} \bar{\vartheta}(t) e^{-r t} d t\right) .
\end{gathered}
$$

Furthermore,

$$
\begin{aligned}
\mathbb{E} & \left(Z^{*}(T)\right)^{2} \\
= & z_{0}^{2} e^{\int_{0}^{T}\left(2 r-\tau(t)^{2}\right) d t}-2 \int_{0}^{T} \bar{\vartheta}(t) \mathbb{E}\left(Z^{*}(t)\right) e^{\int_{t}^{T}\left(2 r-\tau(s)^{2}\right) d s} d t \\
& +\int_{0}^{T}\left[\tau(t)^{2} \kappa(t)^{2}+\sigma_{L}^{2}\right] e^{\int_{t}^{T}\left(2 r-\tau(s)^{2}\right) d s} d t=\alpha \gamma^{2}+\delta,
\end{aligned}
$$

where

$$
\begin{aligned}
\delta= & e^{\int_{0}^{T}\left(2 r-\tau(t)^{2}\right) d t}\left(z_{0}-\int_{0}^{T} \bar{\vartheta}(t) e^{-r t} d t\right)^{2} \\
& +\sigma_{L}^{2} \int_{0}^{T} e^{\int_{t}^{T}\left(2 r-\tau(s)^{2}\right) d s} d t .
\end{aligned}
$$

Substituting (42) and (47) into the objective of problem (15), we have the following theorem.

Theorem 4. Under the optimal solution (19), the optimal value function of problem (15) satisfies

$$
\max _{\theta \in \mathscr{A}\left(z_{0}, X_{0}\right)} \mathbb{E}\left(\lambda Z^{*}(T)-\omega Z^{*}(T)^{2}\right)=\alpha \frac{\lambda^{2}}{4 \omega}+\lambda \beta-\omega \delta,
$$

where $\alpha, \beta$, and $\delta$ are defined by (45), (46), and (48), respectively.

\section{The Optimal Asset-Liability Management Strategy and the Efficient Frontier for Problem (14)}

In this section, we will derive the optimal strategy and the efficient frontier for the asset-liability management problem 
(14). According to Lemma 2, a necessary condition for the optimal solution of problem (15) to attain the optimality of problem (14) at the same time is

$$
\lambda^{*}=1+\left.2 \omega \mathbb{E}\left(Z^{*}(T)\right)\right|_{\theta^{*}}
$$

Substituting the expected terminal net wealth (42) into (50), it can easily obtain

$$
\lambda^{*}=\frac{1+2 \omega \beta}{1-\alpha} .
$$

Then

$$
\begin{aligned}
\gamma^{*} & =\frac{1}{2 \omega(1-\alpha)}+\frac{\beta}{1-\alpha} \\
& =\frac{1}{2 \omega} e^{\int_{0}^{T} \tau^{2}(t) d t}+e^{r T}\left(z_{0}-\int_{0}^{T} \bar{\vartheta}(t) e^{-r t} d t\right), \\
\kappa^{*}(t) & =-e^{r t}\left(z_{0}+\frac{1}{2 \omega} e^{-\int_{0}^{T}\left(r-\tau^{2}(t)\right) d t}-\int_{0}^{t} \bar{\vartheta}(s) e^{-r s} d s\right), \\
t & \in[0, T] .
\end{aligned}
$$

Theorem 5. The optimal asset-liability management strategy for problem (14) is given by

$$
\begin{array}{r}
\theta^{*}(t)=\frac{\tau(t)}{\sigma_{S}}\left[Z^{*}(t)-e^{r t}\left(z_{0}+\frac{e^{-\int_{0}^{T}\left(r-\tau^{2}(t)\right) d t}}{2 \omega}\right.\right. \\
\left.\left.-\int_{0}^{t} \bar{\vartheta}(s) e^{-r s} d s\right)\right],
\end{array}
$$

and the efficient frontier for problem (14) satisfies

$$
\begin{aligned}
\operatorname{Var}\left(Z^{*}(T)\right)= & \frac{e^{-\int_{0}^{T} \tau(t)^{2} d t}}{1-e^{-\int_{0}^{T} \tau(t)^{2} d t}} \\
& \times\left[\mathbb{E}\left(Z^{*}(T)\right)-e^{r T}\left(z_{0}-\int_{0}^{T} \bar{\vartheta}(t) e^{-r t} d t\right)\right]^{2} \\
& +\sigma_{L}^{2} \int_{0}^{T} e^{\int_{t}^{T}\left(2 r-\tau(s)^{2}\right) d s} d t
\end{aligned}
$$

Proof. Substituting $\kappa^{*}(t)$ into (19), we obtain the optimal asset-liability management strategy for problem (14) which is specified by

$$
\begin{array}{r}
\theta^{*}(t)=\frac{\tau(t)}{\sigma_{S}}\left[Z^{*}(t)-e^{r t}\left(z_{0}+\frac{e^{-\int_{0}^{T}\left(r-\tau^{2}(t)\right) d t}}{2 \omega}\right.\right. \\
\left.\left.-\int_{0}^{t} \bar{\vartheta}(s) e^{-r s} d s\right)\right] .
\end{array}
$$

Substituting $\gamma^{*}$ into (42) and (47), the terminal net wealth of the asset-liability management problem (14) under the optimal strategy (53) satisfies

$$
\begin{aligned}
& \mathbb{E}\left(Z^{*}(T)\right)=\gamma^{*} \alpha+\beta=\frac{1}{2 \omega} \frac{\alpha}{1-\alpha}+\frac{\beta}{1-\alpha}, \\
& \mathbb{E}\left(Z^{*}(T)\right)^{2}=\left(\gamma^{*}\right)^{2} \alpha+\delta=\frac{\alpha}{(1-\alpha)^{2}}\left(\frac{1}{2 \omega}+\beta\right)^{2}+\delta .
\end{aligned}
$$

Furthermore, the variance of the terminal net wealth under the optimal asset-liability management strategy (53) satisfies

$$
\begin{aligned}
\operatorname{Var}\left(Z^{*}(T)\right) & =\mathbb{E}\left(Z^{*}(T)\right)^{2}-\left(\mathbb{E}\left(Z^{*}(T)\right)\right)^{2} \\
& =\left(\alpha-\alpha^{2}\right)\left(\gamma^{*}-\frac{\beta}{1-\alpha}\right)^{2}+\delta-\frac{\beta^{2}}{1-\alpha}
\end{aligned}
$$

Notice that

$$
\gamma^{*}=\frac{\mathbb{E}\left(Z^{*}(T)\right)-\beta}{\alpha} .
$$

Substituting (58) into (57), we can have the trade-off between the expectation and the variance of the terminal wealth

$$
\begin{aligned}
& \operatorname{Var}\left(Z^{*}(T)\right) \\
& =\frac{e^{-\int_{0}^{T} \tau(t)^{2} d t}}{1-e^{-\int_{0}^{T} \tau(t)^{2} d t}}\left[\mathbb{E}\left(Z^{*}(T)\right)-e^{r T}\left(z_{0}-\int_{0}^{T} \bar{\vartheta}(t) e^{-r t} d t\right)\right]^{2} \\
& \quad+\sigma_{L}^{2} \int_{0}^{T} e^{\int_{t}^{T}\left(2 r-\tau(s)^{2}\right) d s} d t
\end{aligned}
$$

Then, the proof is completed.

The efficient frontier (54) presents the trade-off between the expected terminal wealth and the risk measured by the variance of the terminal wealth. For a given expected investment return $\mathbb{E}\left(Z^{*}(T)\right)$, the investment risk is calculated by (54). Equation (54) shows that the efficient frontier is a parabola in the variance-mean plane. Since $0<$ $e^{-\int_{0}^{T} \tau(t)^{2} d t}<1$, the investment risk achieves its minimum $\sigma_{L}^{2} \int_{0}^{T} e^{\int_{t}^{T}\left(2 r-\tau(k)^{2}\right) d k} d t$ when the expected terminal net wealth satisfies $\mathbb{E}\left(Z^{*}(T)\right)=e^{r T}\left(z_{0}-\int_{0}^{T} \bar{\vartheta}(t) e^{-r t} d t\right)$. Notice that $\sigma_{L}^{2} \int_{0}^{T} e^{\int_{t}^{T}\left(2 r-\tau(k)^{2}\right) d k} d t>0$; it implies that the investment risk caused by the liability and the incomplete information cannot be completely hedged by the current portfolio.

Corollary 6. The global minimum variance of problem (14) is given by

$$
\operatorname{Var}\left(Z^{\min }(T)\right)=\sigma_{L}^{2} \int_{0}^{T} e^{\int_{t}^{T}\left(2 r-\tau(s)^{2}\right) d s} d t
$$

and the corresponding terminal net wealth satisfies

$$
\mathbb{E}\left(Z^{\min }(T)\right)=e^{r T}\left(z_{0}-\int_{0}^{T} \bar{\vartheta}(t) e^{-r t} d t\right) .
$$


Moreover, the efficient strategy that achieves $\operatorname{Var}\left(Z^{\min }(T)\right)$ is

$$
\begin{array}{r}
\theta^{\min }(t)=\frac{\tau(t)}{\sigma_{S}}\left[Z^{\min }(t)-e^{r t}\left(z_{0}+\frac{e^{-\int_{0}^{T}\left(r-\tau^{2}(t)\right) d t}}{2 \omega}\right.\right. \\
\left.\left.\quad-\int_{0}^{t} \bar{\vartheta}(s) e^{-r s} d s\right)\right],
\end{array}
$$

where $\left\{Z^{\min }(t): t \in[0, T]\right\}$ is the wealth process corresponding to the efficient strategy $\left\{\theta^{\mathrm{min}}(t): t \in[0, T]\right\}$.

\section{Special Cases}

6.1. The Case of No Liability. When there is no liability, $\bar{\vartheta}(t)=$ 0 and $\sigma_{L}=0$. Then the parameters $\beta, \delta$, and $\kappa^{*}(t)$ reduce to

$$
\begin{aligned}
\beta & =z_{0} e^{\int_{0}^{T}\left(r-\tau(t)^{2}\right) d t}, \\
\delta & =z_{0}^{2} e^{\int_{0}^{T}\left(2 r-\tau(t)^{2}\right) d t}, \\
\kappa^{*}(t) & =-e^{r t}\left(z_{0}+\frac{1}{2 \omega} e^{-\int_{0}^{T}\left(r-\tau^{2}(t)\right) d t}\right) .
\end{aligned}
$$

Furthermore, the optimal asset-liability management strategy (53) reduces to

$$
\theta^{*}(t)=\frac{\tau(t)}{\sigma_{S}}\left[Z^{*}(t)-e^{r t}\left(z_{0}+\frac{e^{-\int_{0}^{T}\left(r-\tau^{2}(t)\right) d t}}{2 \omega}\right)\right],
$$

and the efficient frontier satisfies

$$
\operatorname{Var}\left(Z^{*}(T)\right)=\frac{e^{-\int_{0}^{T} \tau(t)^{2} d t}}{1-e^{-\int_{0}^{T} \tau(t)^{2} d t}}\left(\mathbb{E}\left(Z^{*}(T)\right)-z_{0} e^{r T}\right)^{2} .
$$

Letting $\mathbb{S}\left(Z^{*}(T)\right)=\sqrt{\operatorname{Var}\left(Z^{*}(T)\right)}$, we have

$$
\mathbb{S}\left(Z^{*}(T)\right)=\sqrt{\frac{e^{-\int_{0}^{T} \tau(t)^{2} d t}}{1-e^{-\int_{0}^{T} \tau(t)^{2} d t}}}\left(\mathbb{E}\left(Z^{*}(T)\right)-z_{0} e^{r T}\right) .
$$

Obviously, (68) is a straight line with a slop of $\sqrt{(1-\alpha) / \alpha}$ in the $(\mathbb{S}(Z(T)), \mathbb{E}(Z(T)))$ plane. Furthermore,

$$
\mathbb{E}\left(Z^{*}(T)\right)=z_{0} e^{r T}+\sqrt{\frac{1-e^{-\int_{0}^{T} \tau(t)^{2} d t}}{e^{-\int_{0}^{T} \tau(t)^{2} d t}}} \mathbb{S}\left(Z^{*}(T)\right)
$$

is called the capital market line and $\sqrt{\left(1-e^{-\int_{0}^{T} \tau(t)^{2} d t}\right) /\left(e^{-\int_{0}^{T} \tau(t)^{2} d t}\right)}$ is called the price of risk. We have verified that the results (63)-(69) are the same with Elliott et al. [17] when there is no liability, in particular when $\tau(t)$ is a constant $\tau$, that is, the appreciation rate of the risky asset is completely observable at each time and it is constant. We have verified that the results (63)-(69) are the same with Zhou and Li [23] with only one risky asset. In this sense, this paper extends the model in Elliott et al. [17] to the case incorporating liability and the model in Zhou and Li [23] to the case with incomplete information and liability.
6.2. The Case of Complete Information. If the appreciation rates of the risky asset and the liability at each time can be completely observed and are constants, that is, $\mu(t, X(t))=$ $\bar{\mu}(t)=\mu$ and $\vartheta(t, X(t))=\bar{\vartheta}(t)=\vartheta$, then the parameters $\alpha, \beta$, and $\delta$ reduce to

$$
\begin{aligned}
& \alpha=1-e^{-\tau^{2} T}, \\
& \beta=\left[z_{0}+\frac{\vartheta}{r}\left(e^{-r T}-1\right)\right] e^{\left(r-\tau^{2}\right) T}, \\
& \delta=\left[z_{0}+\frac{\vartheta}{r}\left(e^{-r T}-1\right)\right]^{2} e^{\left(2 r-\tau^{2}\right) T}+\frac{\sigma_{L}^{2}}{2 r-\tau^{2}}\left(e^{\left(2 r-\tau^{2}\right) T}-1\right),
\end{aligned}
$$

where $\tau=(r-\mu) / \sigma_{S}$. Hence, the optimal asset-liability management strategy (53) reduces to

$$
\theta^{*}(t)=\frac{\tau}{\sigma_{S}}\left[Z^{*}(t)-e^{r t}\left(z_{0}+\frac{e^{\left(\tau^{2}-r\right) T}}{2 \omega}\right)+\frac{\vartheta}{r}\left(e^{-r T}-1\right)\right],
$$

and the efficient frontier is specified by

$$
\begin{aligned}
& \operatorname{Var}\left(Z^{*}(T)\right) \\
& =\frac{e^{-\tau^{2} T}}{1-e^{-\tau^{2} T}}\left[\mathbb{E}\left(Z^{*}(t)\right)-\left(z_{0}+\frac{\vartheta}{r}\left(e^{-r T}-1\right)\right) e^{r T}\right]^{2} \\
& \quad+\frac{\sigma_{L}^{2}}{2 r-\tau^{2}}\left(e^{\left(2 r-\tau^{2}\right) T}-1\right) .
\end{aligned}
$$

We have verified that the results (70)-(72) are the same as the results in Xie and Li [7] with only one risky asset.

\section{Conclusion}

In this paper, a mean-variance asset-liability management problem with incomplete observable information is investigated. The evolution of the unobservable financial market state is described by a continuous-time finite-state hidden Markovian chain and the appreciation rates of the risky asset and the liability are modulated by this hidden Markovian chain. By using the separation principle, the assetliability management problem with incomplete information is divided into two separable problems: the filteringestimation problem and the stochastic control problem. The stochastic maximum principle is used to derive the analytical optimal asset-liability management strategy and the efficient frontier is also derived explicitly. The explicit expressions of the optimal strategy and the efficient frontier show that the unobservable market information does have significant impact on the optimal asset-liability management strategy and the efficient frontier. And the models and results in Elliott et al. [17], Zhou and Li [23], and Xie and Li [7] can be derived as special cases of this paper.

\section{Conflict of Interests}

The author declares that there is no conflict of interests regarding the publication of this paper. 


\section{Acknowledgments}

This research is supported by Grants of the Humanity and Social Science Foundation of MoE of China (no. 13YJCZH247), the Philosophy and Social Science Programming Foundation of Guangdong Province (no. GD12XYJ06), the State Key Program of National Natural Science of China (no. 71231008), and the Foundation of Guangdong University of Finance (no. 12XJ02-10).

\section{References}

[1] H. Markowitz, "Portfolio selection," Journal of Finance, vol. 7, pp. 77-91, 1952.

[2] W. F. Sharpe and L. G. Tint, "Liabilities-a new approach," Journal of Portfolio Management, vol. 16, pp. 5-10, 1990.

[3] A. Kell and H. Müller, "Efficient portfolios in the asset liability context," Astin Bulletin, vol. 25, pp. 33-48, 1995.

[4] M. Leippold, F. Trojani, and P. Vanini, "A geometric approach to multiperiod mean variance optimization of assets and liabilities," Journal of Economic Dynamics and Control, vol. 28, no. 6, pp. 1079-1113, 2004.

[5] D. Li and W.-L. Ng, "Optimal dynamic portfolio selection: multiperiod mean-variance formulation," Mathematical Finance, vol. 10, no. 3, pp. 387-406, 2000.

[6] M. C. Chiu and D. Li, "Asset and liability management under a continuous-time mean-variance optimization framework," Insurance: Mathematics and Economics, vol. 39, no. 3, pp. 330$355,2006$.

[7] S. X. Xie and Z. F. Li, "Continuous-time optimal portfolio selection with liability," Journal of System Science and Mathematical Science, vol. 27, no. 6, pp. 801-810, 2007.

[8] L. Yi, Z. Li, and D. Li, "Multi-period portfolio selection for asset-liability management with uncertain investment horizon," Journal of Industrial and Management Optimization, vol. 4, no. 3, pp. 535-552, 2008.

[9] S. Xie, Z. Li, and S. Wang, "Continuous-time portfolio selection with liability: mean-variance model and stochastic LQ approach," Insurance: Mathematics and Economics, vol. 42, no. 3, pp. 943-953, 2008.

[10] H. Yao, Y. Lai, and Y. Li, "Continuous-time mean-variance assetliability management with endogenous liabilities," Insurance: Mathematics and Economics, vol. 52, no. 1, pp. 6-17, 2013.

[11] P. Chen, H. Yang, and G. Yin, "Markowitz's mean-variance asset-liability management with regime switching: a continuous-time model," Insurance: Mathematics and Economics, vol. 43, no. 3, pp. 456-465, 2008.

[12] S. Xie, "Continuous-time mean-variance portfolio selection with liability and regime switching," Insurance: Mathematics and Economics, vol. 45, no. 1, pp. 148-155, 2009.

[13] P. Chen and H. Yang, "Markowitz's mean-variance asset-liability management with regime switching: a multi-period model," Applied Mathematical Finance, vol. 18, no. 1, pp. 29-50, 2011.

[14] J. Wei, K. C. Wong, S. C. P. Yam, and S. P. Yung, "Markowitz's mean-variance asset-liability management with regime switching: a time-consistent approach," Insurance: Mathematics and Economics, vol. 53, no. 1, pp. 281-291, 2013.

[15] L. R. Sotomayor and A. Cadenillas, "Explicit solutions of consumption-investment problems in financial markets with regime switching," Mathematical Finance, vol. 19, no. 2, pp. 251279, 2009.
[16] E. Ĉanakoğlu and S. Özekici, "Portfolio selection with imperfect information: a hidden Markov model," Applied Stochastic Models in Business and Industry, vol. 27, no. 2, pp. 95-114, 2011.

[17] R. J. Elliott, T. K. Siu, and A. Badescu, "On mean-variance portfolio selection under a hidden Markovian regime-switching model," Economic Modelling, vol. 27, no. 3, pp. 678-686, 2010.

[18] J. B. Detemple, "Asset pricing in a production economy with incomplete information," Journal of Finance, vol. 41, pp. 383391, 1986.

[19] J. B. Detemple, "Further results on asset pricing with incomplete information," Journal of Economic Dynamics and Control, vol. 15 , no. 3, pp. 425-453, 1991.

[20] M. U. Dothan and D. Feldman, "Equilibrium interest rates and multiperiod bonds in a partially observable economy," Journal of Finance, vol. 41, pp. 369-382, 1986.

[21] G. Gennotte, "Optimal portfolio choice under incomplete information," Journal of Finance, vol. 41, pp. 733-746, 1986.

[22] R. Lipster and A. N. Shiryaev, Statistics of Ramdom Processes, Springer, Berlin, Germany, 2003.

[23] X. Y. Zhou and D. Li, "Continuous-time mean-variance portfolio selection: a stochastic LQ framework," Applied Mathematics and Optimization, vol. 42, no. 1, pp. 19-33, 2000. 


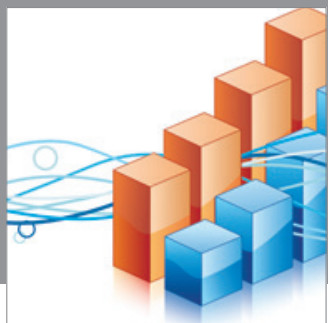

Advances in

Operations Research

mansans

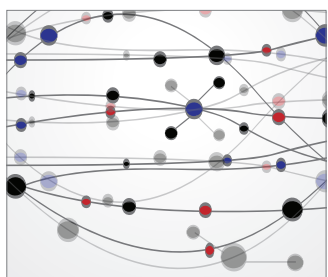

The Scientific World Journal
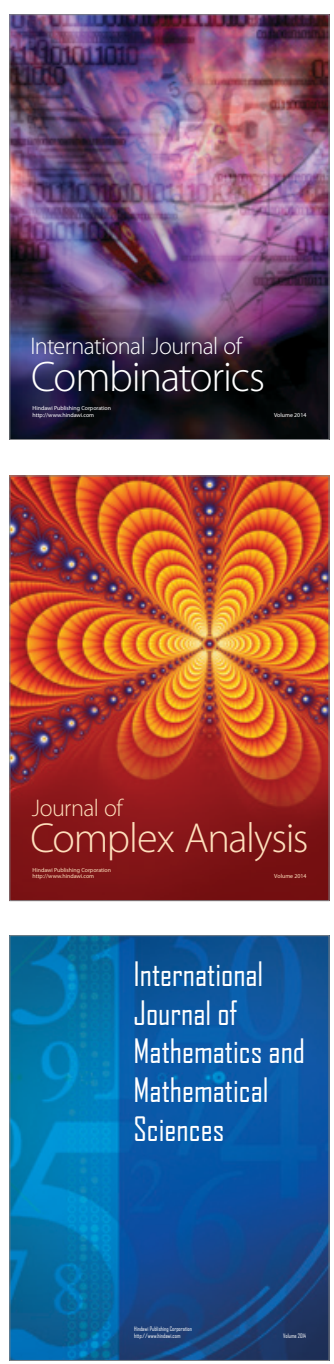
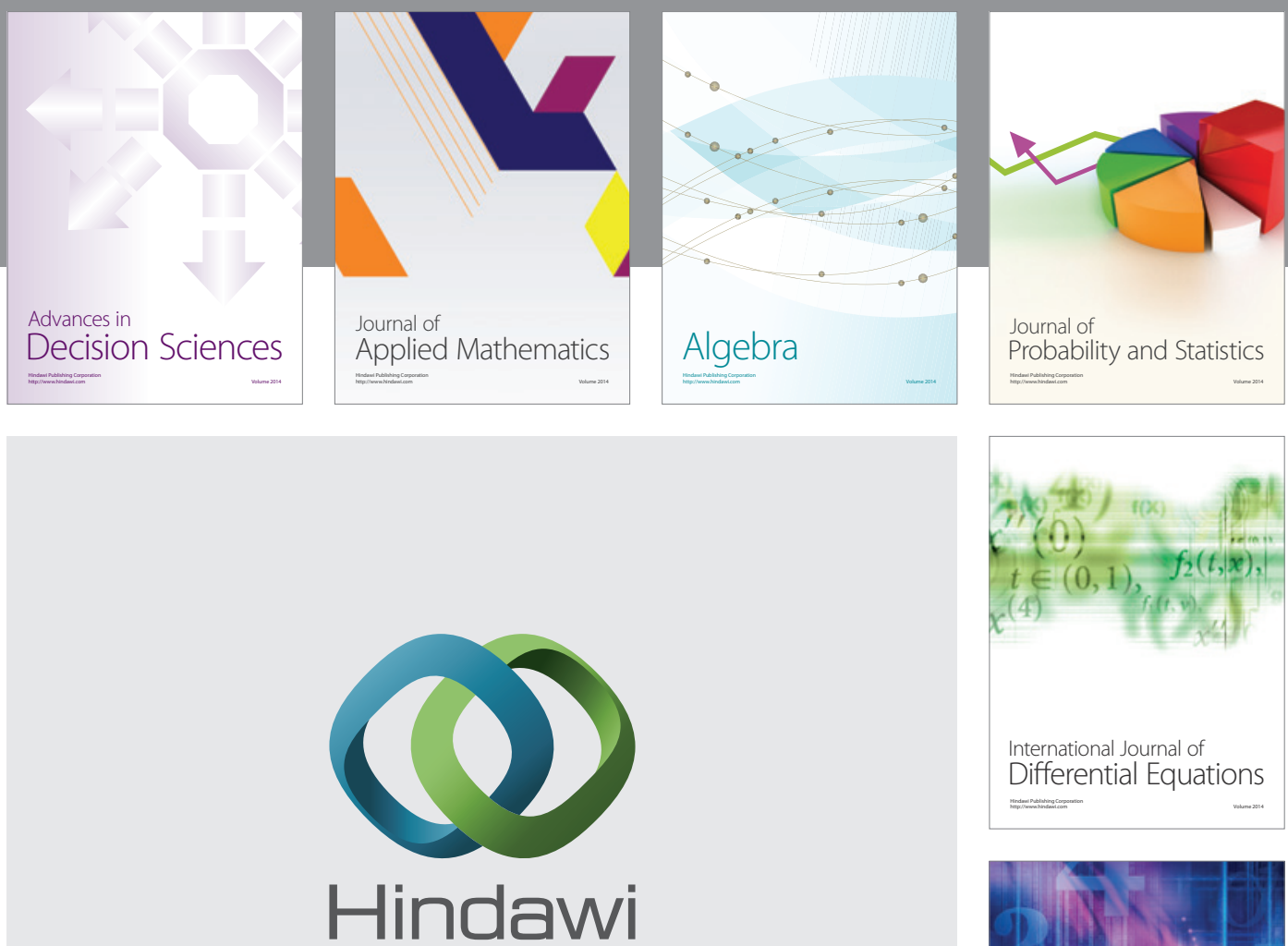

Submit your manuscripts at http://www.hindawi.com
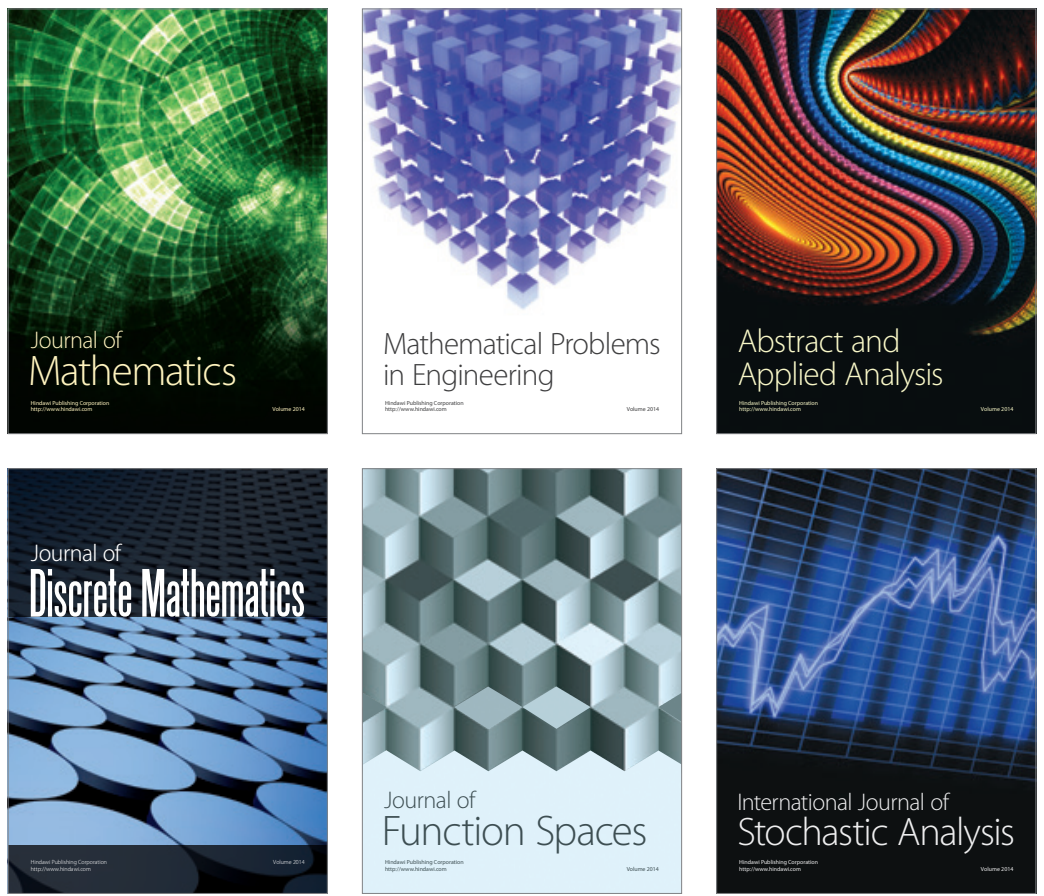

Journal of

Function Spaces

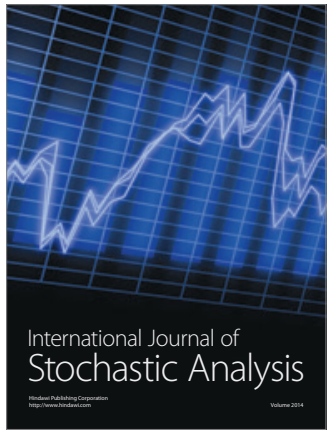

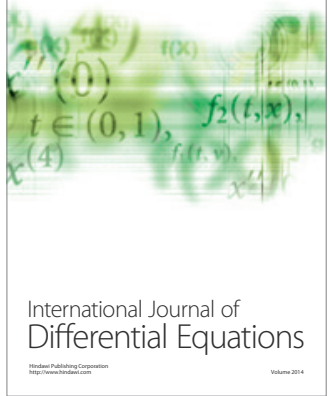
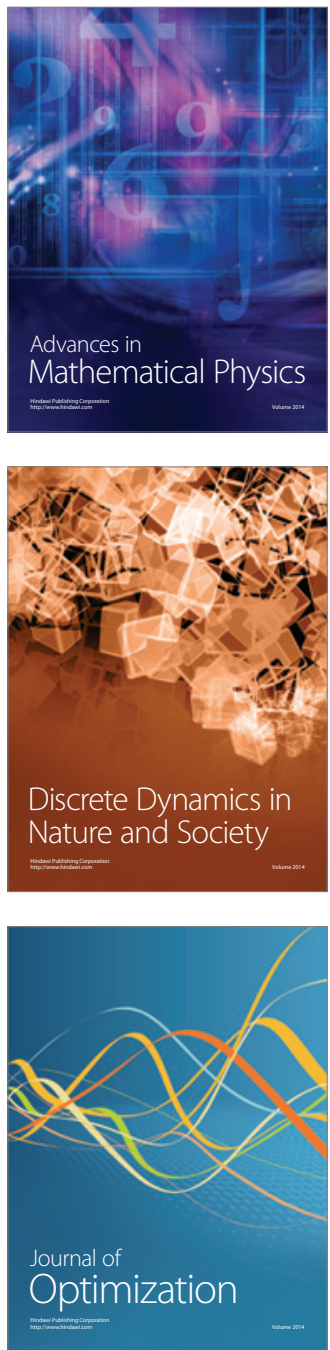